\title{
Exploring Use Cases for Telepresence Robots
}

\author{
Katherine M. Tsui, Munjal Desai, and \\ Holly A. Yanco \\ University of Massachusetts Lowell \\ 1 University Avenue, Lowell, MA 01854 \\ \{ktsui, mdesai, holly\}@cs.uml.edu
}

\author{
Chris Uhlik \\ Google Inc \\ 1600 Amphitheatre Parkway \\ Mountain View, CA 94043 \\ cuhlik@google.com
}

\begin{abstract}
Telepresence robots can be thought of as embodied video conferencing on wheels. Companies producing these robots imagine them being used in a wide variety of situations (e.g., ad-hoc conversations at the office, inspections and troubleshooting at factories, and patient rounds at medical facilities). In July and August 2010, we examined office-related use cases in a series of studies using two prototype robots (Anybots' QB and VGo Communications' VGo). In this paper, we present two studies: conference room meetings $(n=6)$ and moving hallway conversations $(n=24)$. We discuss who might benefit from using telepresence robots, in what scenarios, and the features that telepresence robots must incorporate for use in ad-hoc interactions.
\end{abstract}

\section{Categories and Subject Descriptors}

1.2.9 [Robotics]: Commercial robots and applications

\section{General Terms}

Experimentation

\section{Keywords}

Remote presence, teleoperation, video conferencing

\section{INTRODUCTION}

Despite recent press (e.g., [7, 17, 22]), the concept of telepresence is not new. In 1980, Marvin Minksy painted a picture of people suiting up in sensor-motor jackets to work at their jobs thousands of miles away [19]. He called the remote control tools telepresences, which emphasized the idea of remotely "being there" in such a high fidelity manner that it would seem as though the experience was "in person.'

Thirty years later, how close are we to Minsky's vision? In 2000, the US Food and Drug Administration approved the da Vinci Surgical System by Intuitive Health for laparoscopic surgeries [22]. In his 2009 book, "Wired for War," P. W. Singer wrote about a nineteen year old soldier living in Nevada who flew unmanned

Permission to make digital or hard copies of all or part of this work for personal or classroom use is granted without fee provided that copies are not made or distributed for profit or commercial advantage and that copies bear this notice and the full citation on the first page. To copy otherwise, to republish, to post on servers or to redistribute to lists, requires prior specific permission and/or a fee.

HRI'11, March 6-9, 2011, Lausanne, Switzerland.

Copyright 2011 ACM 978-1-4503-0561-7/11/03 ...\$10.00. aerial vehicles to fight the war in Iraq [23]. In some sense, our progress is close to what Minsky projected, but these are only a small number of highly specialized telepresence systems.

Current telepresence manifests itself in a large number of places in the form of interaction through live video. Friends and family who are located across continents keep in touch with each other through their web cameras and streaming video chat applications such as iChat, Skype, and Google Talk Video, launched in 2003, 2006, and 2008 respectively [1 $116 \mid 25]$. The video conference meeting is a daily activity for some workers. Telepresence through video conferencing ranges from the one-on-one video applications, to dedicated highend telepresence rooms with panoramic displays, to video kiosks designed for the person "dialing in" to embody (e.g., [28]).

Robotics has re-entered the telepresence space, but not as manipulators in sealed nuclear facilities as envisioned by Minsky [19]. Research in the domain of telepresence robots has yielded robots such as robot submarines for subsea exploration [9], the Mars Rovers for space exploration [14], and Geminiod HI-1 for inter-personal communication across distances to better convey a person's remote physical presence [21]. Commercial telepresence robots can be described as embodied video conferencing on wheels. These new telepresence robots provide a physical presence and independent mobility in addition to communication, unlike other video conferencing technologies. InTouch Health was the first to this new communication telepresence robot market with their Remote Presence (RP) robots. In 2003, trials began at rehabilitation centers [10] and eldercare facilities [11], and in hospitals [12] in 2004.

After their commercial launch of the Roomba in 2002 [4], iRobot also approached this new communication telepresence robot space with the consumer in mind. They announced their $\$ 3,500$ CoWorker robot in 2002 and their \$500 ConnectR robot (a Roomba with a video camera) five years later [13]. Neither product caught on, and Colin Angle noted that "off the shelf component costs still have not come down to the point that the business opportunity becomes irresistible" [4]. However, iRobot still believed in the concept of remote presence and the emergence of several new communication telepresence robots (e.g., Anybots' QB, HeadThere's Giraffe, RoboDynamics' TiLR, VGo Communications' VGo, and Willow Garage's Texai) in the last few years has proven them correct.

These companies envision their telepresence robots being used for a wide variety of applications such as ad-hoc conversations at the office, visiting family in eldercare centers, and patient rounds at medical facilities. In July and August 2010, we conducted experiments for several office-related use cases at Google in Mountain View, California, with two telepresence robots: QB and VGo (in beta and alpha testing respectively). Images and technical details are provided in Table 1 In this paper, we present two studies involving conference room meetings and casual hallway conversations while walking. 
Table 1: Key features of the Anybots' QB (left) and the VGo (right) robots used in July and August 2010 studies.

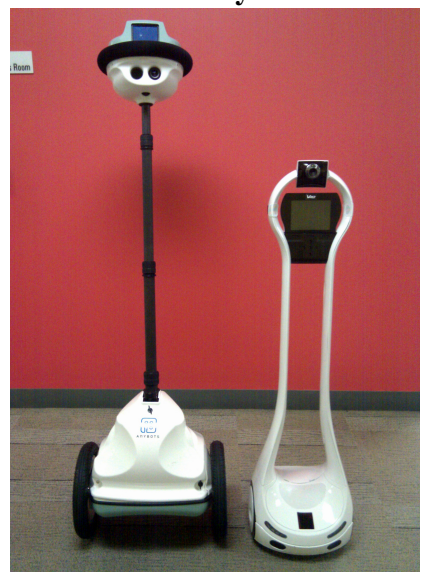

\begin{tabular}{|c|c|c|}
\hline & QB & VGo \\
\hline Unit cost & $\$ 15 \mathrm{~K}$ & $\$ 5 \mathrm{~K}$ \\
\hline Drive & 2 wheels (dynamically balancing) & 2 wheels and 2 casters \\
\hline Top speed & $3.5 \mathrm{mph}$ & $2.75 \mathrm{mph}$ \\
\hline Height & 3'2" to 6'3"' (manually adjusted) & 4 \\
\hline Weight & $35 \mathrm{lbs}$ & $18 \mathrm{lbs}$ \\
\hline Battery life & 4-6 hours & 6 or 12 hour battery option \\
\hline Microphones & 3 on top of head (equally spaced) & 4 around video screen ( 2 front, 2 back) \\
\hline Speakers & 1 on top of head & 2 (woofer in base, tweeter in head) \\
\hline Screen size & 3.5" diagonal & 6" diagonal \\
\hline Number of cameras & 1 front facing and 1 facing down & 1 front facing \\
\hline Camera tilt & no (fixed) & 180 degrees \\
\hline Deictic reference & yes (laser pointer) & no \\
\hline Operating systems & MacOS with Firefox 3.6 & Windows 7/Vista/XP \\
\hline Navigation control & keyboard (arrow keys or WASD) & mouse "Click and Go" or arrows keys \\
\hline 2-way audio & yes & yes \\
\hline 2-way video & no (planned feature) & yes \\
\hline WiFi AP switching & no (planned feature) & yes \\
\hline
\end{tabular}

\section{STUDY 1: FORMAL MEETINGS}

We first examined the conference room meeting. We selected 6 remote participants who had recurring meetings with teammates in Mountain View. The participants, located across the United States and Europe, used the robots to attend their meetings in place of their normal video conferencing setup.

\subsection{Experimental Design}

Our goal was to examine the use of telepresence robots in meetings with different team configurations and different types of teams (i.e., engineering and non-engineering). We chose six teams based on the frequency of their meetings and size of their team (i.e., under 5 people, 5 to 10 people, and over 10 people).

We collected informed consent from the participants who drove the robots (hereafter referred to as "robot drivers") and their teammates who attended the selected meeting. Pre-experiment surveys regarding demographic information, prior robot use, and team dynamics were sent to the robot drivers and their teammates 1 which took approximately 15 minutes to complete. As a baseline, we attended a meeting prior to introducing the robot.

The type of robot assigned to each team (QB or VGo) was dependent upon the type of computer from which the robot driver would use the robot (MacOS or Windows respectively). Table 1 summarizes the key features of the robots. (It should be noted that the QB and the VGo robots were in beta and alpha testing respectively. Both are to be sold starting in Fall 2010.) We trained the robot drivers on the robot they would use to attend the meeting. The robot drivers were asked to fill out a post-training questionnaire regarding their first experience driving the robot and to describe what hardware and software features they liked, disliked, and thought were missing.

We then brought the robots to each robot driver's meeting. We arrived 10 minutes prior to the start of the meeting to conduct a sound check. In each meeting, we performed live-coding of the interactions between the robot drivers and their teammates. The robot drivers were able to continue using the robots up to 30 minutes after the conclusion of the meeting. Prior to the next meeting, we asked the robot drivers if they wished to continue using the robot. We invited the robot drivers to document their use of their robots through a digital diary. At the conclusion of the robot's use, post-experiment surveys regarding team dynamics and the overall experience of the robots were sent to the robot drivers and their teammates. ${ }^{1}$ Additionally, we interviewed the robot drivers.

\footnotetext{
${ }^{1}$ Survey completion was optional for the teammates.
}

\subsection{Participants}

Our six teams had a few different configurations for which we use the terms "hub" and "spoke." A "hub" indicates a group with critical mass. A "spoke" indicates a small number of people (usually 1 or 2) as an offshoot from the hub.

Team 1 was an engineering group. They were a hub-hub configuration with one spoke, meaning that the majority of the team worked in one of two locations. The first hub was located in Mountain View $(n=9)$. The second was located in New York City $(n=14)$. The robot driver was located in Reston, Virginia, and used a QB robot. Team 1 met daily with one weekly 60 minute meeting and four "stand up" meetings approximately 15 minutes in length 2

Team 2 was a non-engineering group. They were a hub-hub configuration with one hub in Mountain View $(n=4)$ and the other hub in London $(n=4)$. The robot driver was located in London and used a VGo robot. Team 2 met weekly for 60 minutes.

Team 3 was an engineering team. They were a hub-spoke-spoke configuration with the hub in Mountain View $(n=18)$, one spoke in Seattle $(n=2)$, and the other spoke in Zurich $(n=2)$. The robot driver was located in Zurich and used a QB robot. Team 3 had weekly 60 minute design review meetings.

Team 4 was a non-engineering team. They were a spoke-spoke configuration, and their entire team was remotely distributed. Team 4 had a weekly one-on-one 60 minute meeting between a manager in Mountain View and his employee in Atlanta. The robot driver used a QB robot.

Team 5 was an engineering team. They were a hub-hub configuration with one hub in Mountain View $(n=13)$ and the other hub in Pittsburgh $(n=8)$. The robot driver was located in Pittsburgh and used a QB robot. Team 5 had a 60 minute weekly meeting.

Team 6 was a non-engineering team. They were configured as a hub-spoke configuration with the hub in Mountain View $(n=7)$. The robot driver, located in Santa Monica, California, used a VGo robot to attend a meeting every two weeks. Due to the infrequency of the meetings, we did not not have time to observe a baseline meeting.

\subsection{Meeting Schedule}

We had planned to use the telepresence robots in a total of thirtysix meetings: twenty-one meetings for Team 1 ; four meetings for Team 4; three meetings for Teams 2, 3, and 5; and two meetings for Team 6. Due to schedule changes described below, the telepresence robots were brought to sixteen meetings. We define a successful

\footnotetext{
${ }^{2}$ The team met in a scheduled conference room and provided quick status updates while standing around the table.
} 
Table 2: Pre-experiment survey examining team dynamics from the perspective of the robot drivers (RD) and their teammates (T). (Grey indicates statistical significance, $p<0.05$ )

\begin{tabular}{|c|c|c|c|c|c|c|c|c|c|}
\hline \multirow[b]{2}{*}{$\begin{array}{l}\text { Scale } \\
\text { Value }\end{array}$} & \multirow[b]{2}{*}{ Item } & \multicolumn{2}{|c|}{ Team 1} & \multicolumn{2}{|c|}{ Team 3} & \multicolumn{2}{|c|}{ Team 4} & \multicolumn{2}{|c|}{ Team 5} \\
\hline & & $\begin{array}{c}\bar{x}_{T}(\mathrm{SD}) \\
(n=7)\end{array}$ & $x_{R D}$ & $\begin{array}{c}\bar{x}_{T}(\mathrm{SD}) \\
(n=2)\end{array}$ & $x_{S D}$ & $\begin{array}{c}\bar{x}_{T} \\
(n=1)\end{array}$ & $x_{R D}$ & $\begin{array}{c}\bar{x}_{T} \text { (SD) } \\
(n=3)\end{array}$ & $x_{R D}$ \\
\hline \multirow{8}{*}{ 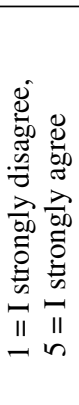 } & $\begin{array}{l}\text { I feel that my remote teammate is ( } \mathrm{I} \text { am) an equal } \\
\text { member of the team. }\end{array}$ & $4.1(0.7)$ & 3 & $4.5(0.7)$ & 2 & 5 & 5 & $4.0(1.0)$ & 5 \\
\hline & $\begin{array}{l}\text { There is good lateral communication with my re- } \\
\text { mote teammate (my team). }\end{array}$ & $3.6(0.8)$ & 3 & $3.0(0.0)$ & 2 & 5 & 4 & $3.3(0.6)$ & 5 \\
\hline & $\begin{array}{l}\text { I trust that my teammates will do their fair share } \\
\text { of the work. [18] }\end{array}$ & $4.1(0.7)$ & 4 & $3.5(0.7)$ & 4 & 5 & 4 & $4.7(0.6)$ & 5 \\
\hline & I enjoy working with my teammates $|\overline{18}|$ & $4.1(0.7)$ & 4 & $4.5(0.7)$ & 4 & 5 & 5 & $4.3(0.6)$ & 5 \\
\hline & I wish I were on a different team [18] & $2.6(1.0)$ & 2 & $3.0(0.0)$ & 3 & 1 & 1 & $3.0(0.0)$ & 3 \\
\hline & The team works well together $[18]$ & $3.7(0.8)$ & 4 & $3.5(0.0)$ & 3 & 5 & 5 & $4.3(0.6)$ & 5 \\
\hline & Everyone contributes to the discussion $|18|$ & $3.4(1.3)$ & 2 & $2.0(0.0)$ & 4 & 5 & 5 & $3.7(1.5)$ & 3 \\
\hline & The team wastes a lot of time $[\overline{18}]$ & $2.6(1.1)$ & 2 & $2.5(0.7)$ & 2 & 1 & 1 & $2.3(0.6)$ & 3 \\
\hline \multirow{7}{*}{ 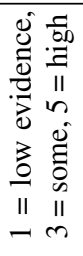 } & Unproductive meetings $[20]$ & $2.0(1.2)$ & 3 & $3.0(0.0)$ & 4 & 1 & 1 & $2.3(1.2)$ & 1 \\
\hline & Communication problems $[20]$ & $2.0(1.2)$ & 3 & $2.5(0.7)$ & 4 & 1 & 1 & $1.3(0.6)$ & 1 \\
\hline & Apathy or lack of involvement $|20|$ & $2.0(1.0)$ & 4 & $1.5(0.7)$ & 4 & 1 & 1 & $2.0(1.0)$ & 2 \\
\hline & Lack of collaboration $|20|$ & $1.7(0.8)$ & 3 & $1.5(0.7)$ & 4 & 1 & 1 & $1.3(0.6)$ & 2 \\
\hline & Lack of trust among team members $|\overline{20}|$ & $1.4(0.8)$ & 3 & $1.0(0.0)$ & 5 & 1 & 1 & $1.0(0.0)$ & 1 \\
\hline & Lack of respect among team members $|20|$ & $1.4(0.8)$ & 3 & $1.5(0.7)$ & 3 & 1 & 1 & $1.0(0.0)$ & 1 \\
\hline & Low attendance $[\overline{20}]$ & $1.7(1.0)$ & 2 & $2.0(0.0)$ & 3 & 1 & 1 & $1.3(0.6)$ & 1 \\
\hline
\end{tabular}

No data was collected from teammates of Teams 2 and 6 or from the Team 6 robot driver. Team 4 did not have enough data for statistical analysis due to only having 1 teammate.

use of the telepresence robots as the robot driver attending his or her meeting primarily through the robot (audio and video) for the duration of the meeting. We observed six successful meetings: Team 1 had three, and Teams 3, 4, and 5 had one each. In the remaining ten meetings, the robot drivers resorted to using video conferencing partway through the meeting (six meetings) or at the start of the meeting (four meetings) due to technical issues with the robots.

The telepresence robots were used for existing meetings. This real-world setting meant that our observations were subject to the meetings being cancelled, teammates going on business travel, and team goals changing. For Team 1, the robot driver had a two week business trip to Asia during which time she did not attend the stand up meetings due to the time zone difference. For Teams 2 and 3, two of the planned three meetings were cancelled by each team.

There was an agenda change for Team 2 given that a teammate from London was visiting Mountain View for one month, resulting in two meetings being cancelled. The robot driver reported this change, and we offered unrestricted use of a robot. A teammate in Mountain View hosted the robot, and the robot driver in London had full access to the robot for one week outside of the originally scheduled usage. The robot driver and teammate used the robot to attend an additional meeting (the researchers were not present).

\subsection{Results}

\subsubsection{Team dynamics}

In the pre-experiment surveys, we asked the robot drivers and their teammates to rate their team dynamics. We used rating items from [18] and [20] and incorporated two of our own statements which examined the equality of the robot driver on the team and the quality of communication with the robot driver (Table 2). The small sample size for each team should be noted, as feedback from the teammates in this study was optional; 7 of 23 teammates from Team 1 responded, 0 of 7 from Team 2, 2 of 8 from Team 3, 1 of 1 from Team 4, 3 of 20 from Team 5, and 0 of 7 from Team 6.

We found that there was a discrepancy between the robot drivers' views and the teammates' views about team cohesion (Table 2). Team 1's robot driver's ratings and teammates' ratings were found to be significantly different for six of the team cohesion items using two-tailed Wilcoxon's signed ranks tests. The most striking difference was found in the statement "I feel like I am (my remote teammate is) an equal part of the team." Team 1's robot driver provided a significantly lower rating $(x=3)$ than her teammates $\left(\bar{x}_{T}=4.1\right.$, $\mathrm{SD}=0.7)(p=0.02, Z=-2.27)$. A similar discrepancy existed in Team 3 as well, but not significantly so due to teammate sample size.

After using the robots, the robot drivers and their teammates were again asked to rate their team's cohesion. Due to the very small sample size (5 responses from teammates), we are unable to determine if there was a change in the team cohesion ratings from the teammate's perspective. Using a series of Wilcoxon's signed ranks tests, we found that there was no significant difference between the robot drivers' responses prior to using the robot and their responses after using the robot. Thus, we found that using the robots did not impact the robot drivers' perspectives which we believe are factors of using the robots for less than a month as well as the reliability of these alpha and beta stage robots.

\subsubsection{Reasons for disuse}

The use of the telepresence robots was allowed by the teammates as long as business as usual could be accomplished. The Team 1 teammates expressed some concern about using the telepresence robot for their meetings. It should be noted that Team 1 had several major deadlines that occurred during the timeline of this study. We introduced the robot ( $\mathrm{a} \mathrm{QB}$ ) during one of their daily stand up meetings. Given the robot driver's and teammates' first experience with the robot, they decided to use the robot only for the stand up meetings as the 60 minute weekly meeting was more critical than the stand up meetings to the team's workflow.

The robot drivers and teammates could stop using the telepresence robots at any time for any reason. As previously mentioned, we asked the robot drivers prior to their next meeting if they would like to continue using the robots. Teams 4 and 5 ended their participation 


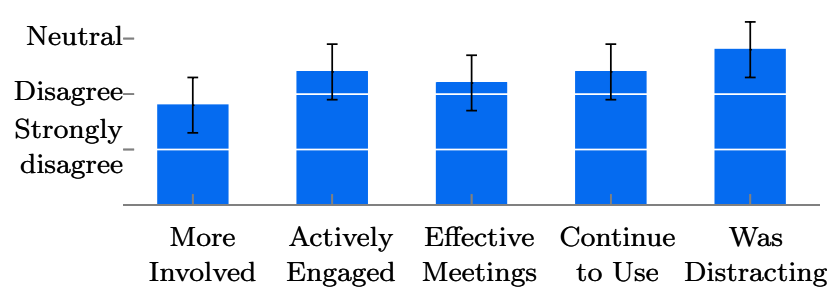

Figure 1: Teammates' overall experiences with the telepresence robots being used in their meetings $(n=5)$. Teammates from Teams 3 and 6 did not report. Note that the vertical axis is truncated due to the largely negative responses.

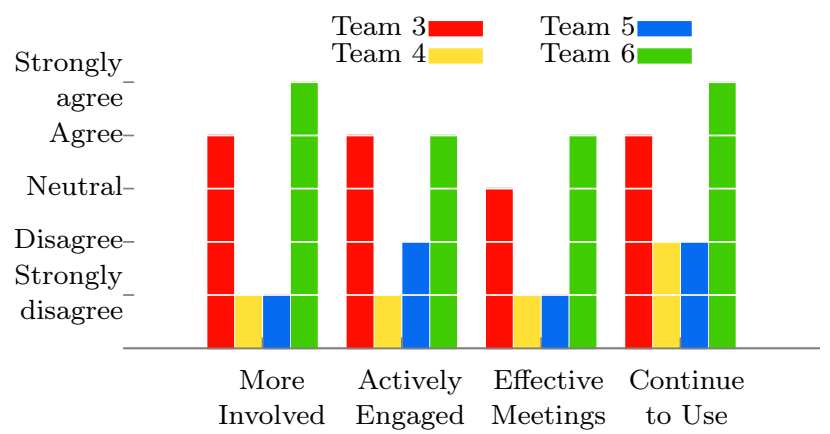

Figure 2: Robot drivers' overall experiences using the telepresence robots to attend in their meetings $(n=4)$. The robot drivers for Teams 1 and 2 did not complete surveys.

in the study prior to completing all of their planned meetings. Team 4 had a weekly one-on-one meeting between a manager in Mountain View and his employee in Atlanta. After their first meeting using the QB robot, we independently asked the robot driver and his manager if they would like to continue. The manager remarked that he would prefer a speakerphone conversation over the robot but would continue to use the robot in the meeting if his employee felt it was beneficial ${ }^{3}$ The robot driver, who was not told about his manager's comments, also desired to discontinue use of the robot. The robot driver for Team 5 ended his participation after completing two of the three meetings, but did not elaborate on his decision.

\subsubsection{Overall experience with the robots in meetings}

We had five teammates from four teams provide responses in the post-experiment questionnaire. On a scale of 1 to 5 ( $1=$ I strongly disagree, $5=$ I strongly agree), the teammates were asked to rate a series of statements including "I feel that my remote teammate is more involved in my team when he/she uses the telepresence robot," "My remote teammate was actively engaged in post-meeting discussions," "The meetings are just as effective if my remote teammate uses the telepresence robot," "I want my remote teammate to continue using the telepresence robot," and "The telepresence robot was distracting in my meetings." The teammates overall reported negative experiences with the telepresence robots being used in their meetings (as shown in Figure 1). Two of the respondents reported preferring their remote teammates (the robot drivers) to use video conferencing instead of the robot. We believe that the responses were due to the technical issues experienced; three of the five respondents reported that the robots were not reliable.

The robot drivers reported a mixture of experiences in some form

\footnotetext{
${ }^{3}$ At the time of this study, the QB robot driver could view live video from the robot's cameras, but the screen on the head of the QB robot was blank. Since this study, the QB robot shows a profile picture of the robot driver. Two-way video is planned.
}

through the post-experiment surveys and interviews. We asked the robot drivers if they would want to continue to use telepresence robots to attend their meetings. Three robot drivers responded positively, two negatively, and the remaining one did not indicate either way if he would continue use. As seen in Figure 2, the robot drivers from Teams 3 and 6 reported more positive experiences. Both robot drivers felt more involved with their teams when they used the telepresence robots and also that they were more actively engaged in post-meeting discussions. In addition to Teams 3 and 6 , the robot driver for Team 1 also reported having a positive experience in her post-experiment interview. Preferences were independent of robot type (Teams 1 and 3 used a QB and Team 6 used a VGo).

We believe that this bimodal grouping was influenced by two factors: their team configuration and their previous work location. In examining the configurations of Teams 1, 3, and 6, we found that the robot drivers were all spoke nodes in a hub-spoke configuration. For Teams 1 and 6, the robot drivers had originally worked at their hub: Team 1's robot driver had been located in New York City with her other 14 teammates, and Team 6's robot driver had been located in Mountain View with his 7 teammates.

\subsection{Discussion}

We believe that telepresence robots will be best used by people who are in hub-spoke team configurations and are in the spoke position, particularly for people who change locations to a spoke after being part of the hub. The Team 1 robot driver noted that, being in a remote office, she knew how important it was for her teammates to feel like they have good quality interaction with her, which is easily accomplished with face to face conversations. The Team 1 robot driver thought that the telepresence robot was a "great way to create those feelings."

After having used the telepresence robots for their recurring meetings, the robot drivers' consensus was that the telepresence robots were not best used for recurring meetings located in conference rooms. The Team 3 robot driver specifically noted that meetings are primarily sitting with no need to move around. We observed that once the robot driver drove into the conference room and into a comfortable position, there were not many large movements. The five robot drivers who attended group meetings moved the robots left and right to indicate attention in the meeting, turning towards the person who was then talking.

The Team 5 robot driver posited that his presence was perhaps more felt when using the telepresence robot, which is supported anecdotally by a report from the Team 6 robot driver. He said, "People asked me a lot more questions. I felt like I was in the room again. [The people in Mountain View] looked at the robot as if it were me. They gestured with their hands towards the robot and made eye contact. They remembered that I was part of the meeting."

The robot drivers offered other use cases which involved more ad-hoc interaction and mobility. The Team 6 robot driver remarked that he wanted an interaction that was more personal than pinging someone (via chat) and suggested that in a distributed office, a telepresence robot could be used to go to a person's cube or office to have a conversation. Another suggested situation was chatting in a hallway. The Team 5 robot driver thought that a telepresence robot could be used in cube or office situations that require interaction such as troubleshooting and looking at someone's screen.

The Team 3 robot driver suggested using a telepresence robot before or after a meeting or when no meeting was scheduled. Twice, the Team 1 robot driver chatted with her teammates via robot prior to their meeting. Her conversations were largely social but included some quick meeting related questions. The Team 3 robot driver used the robot for post-meeting conversation. His meeting ended 10 
minutes early and, as the conference room emptied, the robot driver asked two of his teammates to quickly discuss a matter that had not been resolved completely in the meeting. They continued their conversation until the next meeting's attendees arrived. The robot driver then followed his teammates out to an open common space with couches where the conversation continued another 10 minutes. The Team 3 robot driver remarked that "driving to continue talking was very helpful. If the robot is there at the end of the meeting, then there is no continuity break. It is a different story if the video conference ends and then you need to find another room to continue the conversation." The Team 3 robot driver mentioned that "if the robot were available at any time, then I would do it. Actually, I work quite a bit from home, and would use this all the time."

\section{STUDY 2: HALLWAY CONVERSATIONS}

The importance of examining hallway conversations is highlighted by our Team 3 robot driver from Study 1. As a meeting adjourns, unresolved items can be addressed in the "post-meeting meeting" conversations that occur when walking out the door. Hence the aim of this study was to investigate such an ad-hoc scenario involving movement while simultaneously having a conversation. We felt this was an important scenario that needed to be explored, as mobility is one characteristic that differentiates telepresence robots from video conferencing. We conducted a between-subjects study in which one person operated the robot and had a walking conversation with another person who was physically present with the robot.

This study investigated state-of-the-practice telepresence robots that: 1) are not autonomous entities, 2) have a human-in-the-loop, 3 ) are designed specifically to provide remote presence and, 4) are operating in an uncontrolled real-world environment. Researchers have investigated how autonomous robots collocated with people should navigate in a social setting [6, 24, 27]. Researchers have also investigated different aspects of teleoperating a remote robot such as how much situation awareness an operator has, the design of user interface, and adjustable autonomy for robot behaviors [2, 3, 15]. However, to our knowledge, this study is amongst the first to use commercially available telepresence robots with potential end-users in a real-world setting in which the robots would normally be used.

\subsection{Experimental Design}

We recruited participants and paired them such that the majority did not know each other. The participants met an experimenter in the lobby of the building. One of the participants was randomly chosen to drive the robot first (the "robot driver"). The other participant (the "walker") would drive the robot during the second run. After the participants signed the informed consent forms, they were asked to fill out a pre-experiment questionnaire. The task was holding a conversation with the other person while going to the destination point. The walker and the robot driver were given a list of sample conversation topics.

The robot driver was asked to follow the walker's lead. The walker was given a map which showed the start location, destination point, and 105 foot path connecting them (similar to Figure 3. We chose a kitchen area and a copy machine as our start and destination points (labeled A and B in Figure 3 respectively). We counterbalanced the first run's start location.

Each robot driver was trained on the robot's interface and how to use the robot. The participants were allowed to drive the robot until they felt comfortable driving it. While we did not keep track of the training time for each participant, the training time was typically under two minutes, unless there was a technical problem with the robot. The robot was moved back to the start location, and the run began when the participants moved away from the start.

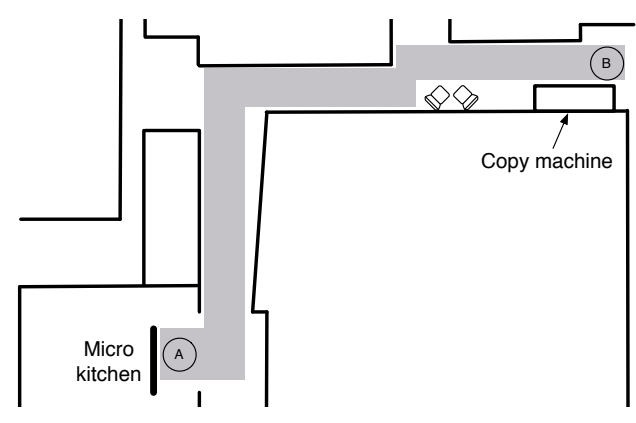

Figure 3: The Study 2 path was approximately 105 feet in length. The narrowest part of the hallway was 6 feet wide. The circles labeled $A$ and $B$ were the end points for the experiment.

There were four termination conditions for the run: 1) if either participant wanted to end the run, 2) the participants reached the destination point, 3 ) the video feed available to the driver paused for more than 30 seconds, or 4) the run exceeded 15 minutes. It should be noted that the VGo robot would switch access points within 20 seconds but the QB robot was unable to switch access points ${ }^{4}$ There were five incomplete runs, but all traversed at least $50 \%$ of the path.

When the run was complete, the participants were asked to fill out a post-task questionnaire which was specific to their role in the run. For the second run, the participants switched roles, and we swapped the start and destination locations. At the end of the second run, the participants were asked to fill out the role-specific post-task questionnaire and also a post-experiment questionnaire. The entire process took 45 minutes on average.

We had 24 participants. There were 11 engineers ( 3 female, 8 male) and 13 non-engineers ( 7 female, 6 male). The average age was 30 years $(S D=8.54)$. All the participants were competent at using a computer; 9 participants assessed their computer expertise as guru, 8 as expert, and 7 as moderate. The participants were not asked about their prior robot experience in the questionnaires. We later asked them via email if they had any prior robot experience. Of the 16 participants that responded, 12 reported having no prior robot experience, 3 reported using Lego Mindstorms, and 1 reported owning a Roomba robot.

There were a total of 13 pairs of runs (7 pairs with QB and 6 with VGo). For two sessions, one participant did not show up. The experimenters substituted for the missing roles but did not fill out questionnaires. For those runs, one experimenter drove the robot while the participant walked next to the robot and then the other experimenter acted as the walker as the participant drove the robot.

\subsection{Results and Discussion}

\subsubsection{Visibility of the walker's face}

An important aspect of having a one-on-one conversation is the ability to look at the other person's face to show engagement and attention. We examined the screen capture videos to see how often the robot driver was looking at the walker's face at least half of the time. We classified the face as being visible if more than half the face appeared in the camera's view. Using the QB robot, the walker's face was visible over $50 \%$ of the time in 6 of 12 runs, not including the two runs in which an experimenter substituted as the robot driver. However for the VGo robot, we found that the walker's face was visible over $50 \%$ of the time in only 2 of 11 runs 5

\footnotetext{
${ }^{4}$ Since the time of this study, VGo Communications has developed faster access point switching. Anybots is also addressing the ability of switching access points.

${ }^{5}$ Only a portion of one of the VGo runs was recorded, so it was not included in this analysis.
} 
We believe that there were two main factors that contributed to only two VGo drivers looking at their walkers' faces: the field of view and the height. The VGo robot's camera is mounted 4 feet from the floor. If a walker with height of 5' 9" was in the robot's personal space (1.5 to 4 feet [8]) or near social space up to 5 feet, then only the walker's body would be visible in the camera's field of view unless the robot driver tilted the camera up. Given the narrow field of view, the robot driver would no longer be able to see the floor ahead which was essential for the simultaneous navigation required for this task.

\subsubsection{Walking position}

For fifteen runs, we noted the walker-robot configuration. Many walkers began with a side-by-side configuration at the 3 or 9 o'clock positions (with the robot driving forward at the 12 o'clock position). Only one participant remained exclusively at the 3 o'clock position. The remaining fourteen either walked directly in front of the robot or to the forward side of the robot. Walkers experimented with a few positions. At some point in their run, thirteen participants walked in front of the robot at the 10,11, or 1 o'clock position. Four participants walked directly in front of the robot with their back to the robot. Two participants walked directly in front of the robot backwards such that they were face to face with the robot.

The difficulty for the walkers was that if they walked side-byside with the robot, as two people would, they were outside of the robot's camera view. Many walkers made the choice to move ahead of the robot so that they were in view of the camera. Unless the walkers walked backwards, they then had to turn their torsos and heads towards the robot to see the video of the robot driver (for the participants using a VGo robot) or the "face" of the robot (for QB).

The distance between the walker and the robot ranged from 2 feet up to 10 feet in one extreme case. It was important for the robot driver to know where the walker was relative to the robot at all times. The QB robot had an auxiliary downward facing camera. The orientation and the field of view of the camera allowed the drivers to see the floor area around the robot, so the driver could potentially know where the walker was even if he or she was out of the front camera's field of view. For 10 out of 12 runs, the walker's feet were visible in the bottom camera over $90 \%$ of the time.

As the VGo robot only had one camera, there were only 6 of 11 runs in which the walker's body was visible to the driver over $50 \%$ of the time. Two participants drove the robots such that the walkers were just on the edge of the camera's field of view. Both of these robot drivers missed the 90 degree turn and their walkers had to intervene to indicate the correct path.

\subsubsection{Not like walking and talking with a person}

We asked the participants to rate how well their conversation through the robot compared to conversing with a person $(1=$ not at all like walking and talking with a person, $5=$ very much like). From the perspectives of both the robot drivers and walkers, the walking hallway conversation was not comparable $(p<0.035$ using a $\chi^{2}$ test). Nineteen robot drivers and thirteen walkers rated the conversation likeness at or below the mode (2). We believe that this unnaturalness is in part due to the height and speed of the robot. The robot drivers rated VGo as moving slower than they would like ( $\bar{x}=1.3, \mathrm{SD}=0.5)$ compared to $\mathrm{QB}$, which was rated as having an appropriate speed $(\bar{x}=1.9, \mathrm{SD}=0.7)(p=0.013$ using a two-tailed Wilcoxon-Mann-Whitney test). The walkers reported that VGo was shorter than they would like ( $\bar{x}=1.3, \mathrm{SD}=0.5)$ compared to $\mathrm{QB}$, which was rated as having an appropriate height $(\bar{x}=1.8, \mathrm{SD}=0.4)$ ( $p=0.016$ using a two-tailed Wilcoxon-Mann-Whitney test).

Other potential reasons for the unnaturalness of the conversation may be due to technical issues with the audio, the driving style, and

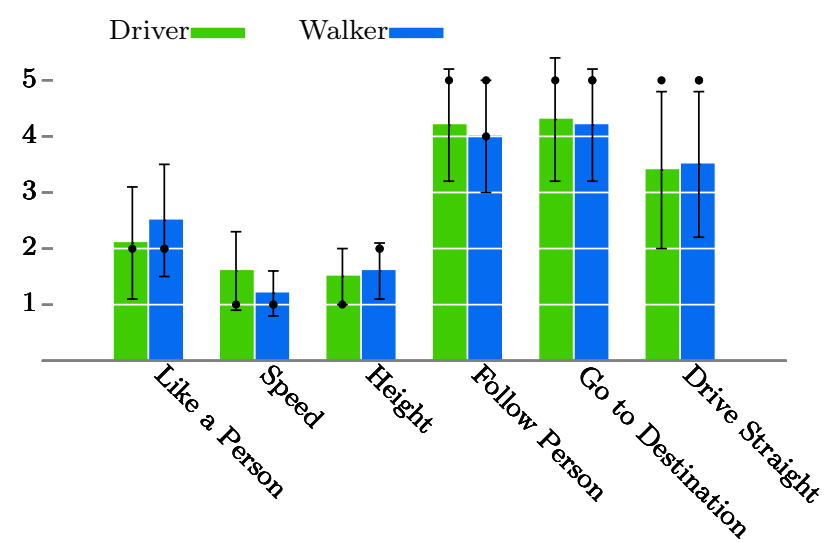

Figure 4: Subset of post-task survey responses for robot drivers and walkers $(n=24)$. The dot represents the mode. Computation of $\chi^{2}$ test found that all responses except ratings for the usefulness of an autonomous drive straight robot behavior to be significant $(p<0.03)$.

the walking position. When people walk together and have a conversation, the pace is fairly constant and the trajectory is smooth unless the hallway is crowded. This human-likeness was infrequently observed for the robots. Only two participants were able to drive the robot continuously using QB and none with VGo. As previously mentioned, the walking position was not side-by-side, as people would be, in this use case. In order for the walker to be in the robot's field of view or see the video of the driver (VGo only), the walker had to walk slightly ahead of the robot.

The most important component of a hallway conversation is the conversation itself. The robots had several audio issues including echo, feedback, and cut outs. The robot drivers could hear themselves talking through the system (QB: 3 of 13 runs, VGo: 8 of 11). There was also feedback through the laptop (QB: 7 of 13 runs, VGo: 2 of 11). In 4 of 13 runs using the QB robot, the audio stream that the driver received was missing parts of sentences. There were no runs in which the VGo robot had choppy audio. These audio issues make it difficult to have any conversation, let alone a natural conversation.

\subsubsection{Need for autonomy}

We asked the robot drivers to rate how much attention they were able to pay to the driving task $(1=$ little attention, $5=$ adequate attention) $\left(\bar{x}=3.9, \mathrm{SD}=0.8, p=0.003, \chi^{2}(4)=16.417\right)$. We also asked the participants about the distribution of attention between the two tasks $(1=$ talk task, $5=$ driving task $)$. Since the use case required the participants to split their attention, the robot drivers reported giving significantly more attention to the driving task $(p<0.001$, $\left.\chi^{2}(4)=20.583\right)$. They noted that they were able to pay more attention to the conversation using the $\mathrm{QB}$ robot $(\bar{x}=3.8, \mathrm{SD}=1.0)$ than the VGo robot $(\bar{x}=3.0, \mathrm{SD}=1.3)$ but not significantly so. We feel this disparity in attention distribution between QB and VGo could be related to the fact that QB had two cameras that allowed the drivers to view the immediate surroundings of the robot and the path straight ahead at the same time. VGo only had one camera that could be tilted up and down by the driver.

The uneven attention distribution could be addressed by adding autonomous navigation behaviors to the telepresence robots. In the post task questionnaire, the participants were asked to rate how useful they thought "Drive straight," "Follow a person," and "Drive to a specific destination" autonomy modes would be ( 1 = not useful, $5=$ very useful). The autonomy modes were listed as is and no additional information regarding the behavior of these autonomy modes was provided. The experimenters were present while the participants answered the questionnaires and they did not receive any 
questions regarding the autonomy modes. From the perspective of both the robot driver and the walker, a follow person behavior and a go to destination mode were rated as potentially quite useful $(p<0.01$ using a $\chi^{2}$ test). Using two-tailed Wilcoxon-Mann-Whitney tests, the drive straight autonomy mode was judged to be more useful for the VGo robot $(\bar{x}=4.0, \mathrm{SD}=1.3)$ than the $\mathrm{QB}$ robot $(\bar{x}=2.8, \mathrm{SD}=1.3)$ $(p=0.037){ }^{6}$ Because of the lag and the dynamic environment, it was difficult to keep the robots driving straight.

In this study, the conversation was first-time acquaintance small talk. It would be difficult to imagine conducting a substantial conversation that involves technical information. However, we believe that the walking hallway conversation is a viable use case despite the number of points mentioned above which indicate how unnatural the current interaction is. With autonomous navigation behaviors, a driver could indicate which person to follow and continue the discussion while in transit to the person's next meeting or moving back to the office.

\section{DESIGN GUIDELINES}

Some guidelines based on the observations made by the experimenters and the results of the studies are presented below 7 These guidelines should be useful in designing mobile telepresence robots.

Autonomous behaviors that can follow a person, navigate to a specified destination, and actively avoid obstacles should be implemented. Driving a remotely operated robot is a challenging task and can overwhelm even experienced operators. Studies about teleoperating robots with more sophisticated sensors highlight some of these issues (e.g., [29]). Providing autonomous behaviors will reduce the cognitive load on the users and allow them to focus more on the conversation, such as a scenario in which the driver is controlling the robot and also conversing with someone. Autonomous behaviors can also be used to keep pace with the person walking next to a robot.

Localization information should be provided to improve the driver's situation awareness about the robot's surroundings. Providing a map of the environment along with the current location of the robot will provide a point of reference and make it easier to navigate in both known and unknown environments.

An independently articulated "head" or torso should be provided. The video screen and the camera for conversation must be able to move independently of the robot's main body. An articulated head or torso would will allow the driver to look at the person walking next to the robot, which would consequently allow the walker to maintain a natural posture and pose when conversing with the driver. If an independent torso cannot be provided, at the very least independent cameras for navigation and conversation should be provided. This camera configuration will allow the driver to navigate using one camera and carry on a conversation using the other camera.

Platform independent user interface (UI) should be used to control the robots. A platform independent UI allows a larger community of users to access the robots. Providing a web-based UI is also recommended as it allows the users to control the robots from any machine at their disposal without needing specific software applications.

Dynamic volume control for robots is necessary since the robots will be operating in quite meeting rooms and offices to noisy public areas. The volume for the speakers on the robot should change

\footnotetext{
${ }^{6}$ The alpha-stage VGo robots used in this study had mismatched gears that caused the robot to drift in one direction at high speeds and the casters bound when they turned. Over time, the hole for the caster's post enlarged due to the soft plastic wearing. Since the study, the drift issue has been addressed and the VGo production units from beta onwards use a stronger material for the casters.

${ }^{7} \mathrm{~A}$ detailed list of guidelines will be published later. Those guidelines will be available at http://robotics.cs.uml.edu/
}

accordingly or let the driver or the people interacting with the robot change the volume.

While these are amongst the first set of design guidelines for telepresence robots, the concepts expressed are not new. Optimized algorithms and implementations for navigation behaviors like obstacle avoidance and path planning already exist (e.g., [5]). Models of how people walk with autonomous robots have also been developed (e.g., [6 26]). While the solutions to some of the issues with current telepresence robots exist, the challenge will be integrating the solutions together such that the effects of out-of-the-loop syndrome are minimal for the robot driver.

\section{THE FUTURE}

Through the series of studies we conducted with the QB and VGo telepresence robots, we gained several insights about office-related use cases. We believe that telepresence robots are well suited for use by remote workers who are part of a spoke in a hub-spoke configuration. All three robot drivers from Study 1 who were spokes in a hub-spoke configuration gave positive comments about their experiences using the robots in meetings. For two of these three participants, they spent some portion of their week working from home. Thus, we believe that the telepresence robots will also provide good experiences for remote workers who work from home. Willow Garage's Dallas Goecker has been telecommuting nearly every day to work with his teammates in Menlo Park, California, via Texai robot from his home in Seymour, Indiana, for over one year [7].

Another interesting commonality between two of the Study 1 robot drivers was that they had originally been part of the hub side of the team. We were approached by a team who recently had a coworker move from Mountain View to another location and missed having coffee with her. They had seen the robots roaming around the building and requested to borrow one robot for a quick 20 minute Monday morning chat. This request emphasizes people's desires to keep their remote teammates involved.

We believe that telepresence robots do have a role to play in an office environment but perhaps not in the context of a formal meeting. In an online survey using Amazon's Mechanical Turk, 75 participants ranked large meetings (greater than 10 people) as one of their top 10 (from a list of 50) situations in which to use telepresence robots 8 Our observations of large meetings in Study 1 indicate otherwise. The most compelling example was found in Team 5. The robot driver had large weekly meetings; however, the robot driver did not feel compelled to use the robot in this situation and terminated his participation early. We believe that the hub-hub configuration of the team may have played a part in his decision. Instead of using the robot in large formal meetings, it may have been more beneficial to assign a telepresence robot to the team in each hub to act as a portal. Two people from Dublin, Ireland, requested a setup in which the telepresence robot "lives" in their remote teammates' cube area.

If telepresence robots were to be used in meetings, then the small, less formal meetings like stand up meetings seem to be a good fit. For example, one participant mentioned that he would use it daily for his stand up meetings. He splits his time equally between Mountain View and San Francisco, and his stand up meetings occur in both locations. He noted that when he is in one location, he is unable to attend the stand up meeting in the other location.

Overall, Study 1 showed the importance of informal interactions before and after the meetings (Team 1 and Team 3 respectively). We believe that hallway conversations are the bridge between conference room discussions and wherever the next location is (e.g., another conference room, common area, cube, etc.). When video conferencing is tied to a particular room, the discussion either has

\footnotetext{
${ }^{8}$ A copy of the survey can be found at $g \circ 0.9 l / w H m Q R$.
} 
to pause until a new location is found or moves to another communication medium (typically email). Using a telepresence robot has the potential to be just as awkward if the remote person has to go "find a body" and come back to the room in order to finish the conversation. However, if there are conference rooms clustered together, it might be desirable to house a few robots close by to reduce retrieval time.

Our studies have focused primarily on the robot driver's perspective and experience. In the future, we will would like to investigate how interpersonal relationships between the robot driver and the people who were physically present with the robot change over time. We would also like to investigate how telepresence robots are perceived by general office personnel (i.e., bystanders) with respect to acceptance, usefulness, and privacy.

\section{ACKNOWLEDGMENTS}

This research has been funded in part by NSF (IIS-0905228, IIS-0546309). We would like to acknowledge Anybots and VGo Communications for loaning us prototype robots. The opinions expressed in this paper are that of the UMass Lowell researchers and not of Google.

\section{REFERENCES}

[1] Apple. Apple Introduces iChat AV and iSight. Press release, June 2003. http://www.apple.com/pr/library/2003/ jun/23ichat.html, accessed Sept. 2010.

[2] D. Bruemmer, J. Marble, D. Dudenhoeffer, M. Anderson, and M. McKay. Mixed-Initiative Control for Remote Characterization of Hazardous Environments. In Proc. of Annual Hawaii Intl. Conf. on System Sciences, page 9, 2003.

[3] J. Drury, H. Yanco, and J. Scholtz. Using Competitions to Study Human-Robot Interaction in Urban Search and Rescue. Interactions, 12(2):39-41, 2005.

[4] W. B. Fox. I, Robot Entrepreneur. America First Books, 2005.

[5] B. Gerkey, R. Vaughan, and A. Howard. The Player/Stage Project: Tools for Multi-Robot and Distributed Sensor Systems. In Proc. of Intl. Conf. on Advanced Robotics, pages 317-323, 2003.

[6] R. Gockley, J. Forlizzi, and R. Simmons. Natural Person-Following Behavior for Social Robots. In Proc. of Intl. Conf. on Human-Robot Interaction, pages 17-24, 2007.

[7] E. Guizzo. When My Avatar Went to Work. IEEE Spectrum, Sept. 2010. http://spectrum.ieee.org/robotics/industrialrobots/when-my-avatar-went-to-work/, accessed Sept. 2010.

[8] E. Hall. The Hidden Dimension. Doubleday New York, 1966.

[9] B. Hine, C. Stoker, M. Sims, D. Rasmussen, P. Hontalas, T. Fong, J. Steele, D. Barch, D. Andersen, E. Miles, et al. The Application of Telepresence and Virtual Reality to Subsea Exploration. In Proc. of Workshop on: Mobile Robots for Subsea Environments, Intl. Advanced Robotics Program, pages 117-126, 1994.

[10] InTouch Technologies, Inc. InTouch Health and Rehabilitation Institute at Santa Barbara Collaborate to Evaluate World's First Mobile Remote Presence Robot for Healthcare. Press release, Jan. 2003. http://www.intouchhealth.com/ pr1-15-03.html, accessed Sept. 2010.

[11] InTouch Technologies, Inc. World's First Remote Presence Robot Used by Healthcare Experts in Elder Care Begins Clinical Testing. Press release, Feb. 2003. http://www. intouchhealth.com/pr2-24-03.html, accessed Sept. 2010.

[12] InTouch Technologies, Inc. Robots at Sentara General Hospital Boosting Doctors' Efficiency. Press release, Apr.
2004. http://www.intouchhealth.com/pr4-27-04.html, accessed Sept. 2010.

[13] iRobot. iRobot Unveils Two New Robots at DigitalLife. Press release, Sept. 2007. http://www.irobot.com/sp.cfm? pageid=86\&id=366\&referrer=169, accessed Sept. 2010.

[14] E. Kac. Editorial: Live from Mars, 1998.

[15] B. Keyes, M. Micire, J. Drury, and H. Yanco. Improving Human-Robot Interaction through Interface Evolution. Human-Robot Interaction, Daisuke Chugo (Ed.), pages 183-202, 2010.

[16] S. Lachapelle. Talk face-to-face right from within Gmail. Official Google Blog, Nov. 2008. http://googleblog. blogspot.com/2008/11/talk-face-to-face-right-fromwithin.html, accessed Sept. 2010.

[17] J. Markoff. The Boss Is Robotic, and Rolling Up Behind You. The New York Times, Sept. 2010. http://www.nytimes.com/ 2010/09/05/science/05robots.html?_r=1, accessed Sept. 2010.

[18] M. Michalisin, S. Karau, and C. Tangpong. The Effects of Performance and Team Cohesion on Attribution: A Longitudinal Simulation. J. of Business Research, 57(10):1108-1115, 2004.

[19] M. Minsky. Telepresence. Omni, 2(9):45-52, 1980.

[20] J. Osland, D. Kolb, I. Rubin, and M. Turner. Organizational Behavior: An Experiential Approach. Prentice Hall Englewood Cliffs, New Jersey, $8^{\text {th }}$ edition, 2007.

[21] D. Sakamoto, T. Kanda, T. Ono, H. Ishiguro, and N. Hagita. Android as a Telecommunication Medium with a Human-like Presence. In Proc. of Intl. Conf. on Human-Robot Interaction, pages 193-200, 2007.

[22] E. Singer. The Slow Rise of the Robot Surgeon. MIT Technology Review, March 2010. http://www.technologyreview.com/biomedicine/24850/?a=f, accessed Sept. 2010.

[23] P. Singer. Wired for War: The Robotics Revolution and Conflict in the Twenty-first Century. Penguin Pr, 2009.

[24] E. Sisbot, R. Alami, T. Simeon, K. Dautenhahn, M. Walters, and S. Woods. Navigation in the Presence of Humans. In Intl. Conf. on Humanoid Robots, pages 181-188, 2006.

[25] Skype. Skype Introduces Video Calling for Macintosh Users. Press release, Sept. 2006. http://about.skype.com/2006/09/ skype_introduces_video_calling.html, accessed Sept. 2010.

[26] E. Sviestins, N. Mitsunaga, T. Kanda, H. Ishiguro, and N. Hagita. Speed Adaptation for a Robot Walking with a Human. In Proc. of Intl. Conf. on Human-Robot Interaction, pages 349-356, 2007.

[27] E. Topp and H. Christensen. Tracking for Following and Passing Rersons. In Intl. Conf. on Intelligent Robots and Systems, pages 2321-2327, 2005.

[28] G. Venolia, J. Tang, R. Cervantes, S. Bly, G. Robertson, B. Lee, and K. Inkpen. Embodied Social Proxy: Mediating Interpersonal Connection in Hub-and-Satellite Teams. In Proc of Intl. Conf. on Human Factors in Computing Systems, pages 1049-1058, 2010.

[29] H. Yanco, J. Drury, and J. Scholtz. Beyond usability evaluation: Analysis of human-robot interaction at a major robotics competition. Human-Computer Interaction, 19(1):117-149, 2004. 